\section{Near-Field Optical Microscope Enables Submicron Direct-Write Laser Micromachining of Diamond}

Diamond came to the attention of the scientific community as a material that could be useful in microelectronic and microelectromechanical systems applications because of its mechanical, electrical, thermal, and chemical properties. However, diamond is very difficult to work with, due to its hardness. Igor Smolyaninov and Christopher Davis from the Department of Electrical and Computer Engineering of the University of Maryland have developed a technique for microscale processing. Using a standard scanning near-field optical-shear-force microscopy technique, they achieved high-resolution surface ablation of thick chemical-vapor-deposition-(CVD)-grown diamond films.

As reported in the October 1 issue of Optics Letters, their method uses nanosecond optical pulses from a Nd:YAG laser operating at 1060-nm wavelength coupled to a multimode optical fiber with an initial core diameter of $150 \mu \mathrm{m}$ adiabatically tapered to a 100-nm microscope tip and used as a local light source. This arrangement allowed them to deliver a power of $\sim 100 \mathrm{MW} / \mathrm{cm}^{2}$ to a sample region of only $500 \mathrm{~nm} \times 500 \mathrm{~nm}$ during a single laser pulse. This power was sufficient for ablating locally the surface of diamond. The ablation process required multiple laser pulses in a given location and may be mediated by an intermediate step in which the diamond is turned into graphite before being ablated. Patterns written on samples were investigated with a shearforce microscope, which was an intrinsic part of their system. Extended use of the optical fiber showed no damage to the tip from either exposure to the laser light or accumulation of graphite. Calculations indicate that the optimum tip diameter is $\sim \lambda / 3$. Thus, even though the diameter for single spots obtained was $\sim 500 \mathrm{~nm}$, the spatial resolution of this micromachining method can be further increased to $50-100 \mathrm{~nm}$ by using a pulsed UV laser.

Claudiu Muntele

\section{Electromagnetic Tweezers Enable Simultaneous Positioning and Viewing of Micron-Sized Magnetic Objects}

The advent of nanotechnology has created a need for techniques that are capable of precisely manipulating and positioning objects that range in size from atomic to micrometer dimensions. Optical trapping methods and microelectromechanical systems (MEMS) have been used for manipulating micron-scale objects of biological interest. For magnetic materials, magnetic tweezers, consisting of permanent or soft coil-wound magnets with macroscopic dimensions, have been employed. Recently, a magnetic material manipulation technique that integrates a low profile magnetic coil and soft ferromagnetic probe has surfaced. This device, which is physically much smaller than other magnetic or optical tweezers, provides several advantages over these other devices, including negligible sample heating, low cost, ability to study particles suspended in a variety of solutions, and simultaneous positioning and optical viewing of samples.

In the September 17 issue of Applied Physics Letters, Mladen Barbic and coworkers of the University of CaliforniaSan Diego described a scanning probe electromagnetic tweezer system that utilizes a microcoil and a magnetic microtip for localized positioning of micron-sized magnetic objects. Their technique also incorporates an optical illumination scheme that allows the magnetically trapped objects to be viewed without the interference that often arises due to light scattering from the magnetic manipulator tip. The device can position magnetic objects with submicron resolution up to operating distances of more than $40 \mu \mathrm{m}$.

The scanning probe electromagnetic tweezers were fabricated by winding 25$\mu \mathrm{m}$-diameter copper magnet wire around a $50-\mu \mathrm{m}$-diameter soft-ferromagnetic wire (two coil layers with 6-8 turns each). The soft-ferromagnetic wire was electrochemically etched into a sharp probe in aqueous $40 \%$ sulfuric acid solution at $3 \mathrm{~V}$. In addition to keeping the device small, by minimizing the coil diameter and the ferromagnetic tip size, the researchers gained in magnetic field strength and field gradient, respectively. The microcoil and microtip, which measure $1 \mathrm{~mm}^{3}$ in size (see figure), were installed on a Nikon Diaphot inverted optical microscope such that the microtweezers tip rested on a mechanical translation stage above the microscope's viewing lens.

Superparamagnetic, $2.8-\mu \mathrm{m}$-diameter beads and $1-\mu \mathrm{m}$-diameter (nonmagnetic) polystyrene (PS) beads were placed inside a rectangular cross-section quartz capillary tube with inner dimensions of $50 \mu \mathrm{m} \times$ $500 \mu \mathrm{m}$ and a uniform wall thickness of $40 \mu \mathrm{m}$. The capillary tube was placed between the tip and the microscope lens, and the microtip was brought to within several microns of the outer capillary wall. White light from a xenon lamp was coupled to the capillary tube through an optical fiber to allow for optical viewing. The capillary holder prevented spurious light scattering from the microtip. Due to differences in the index of refraction

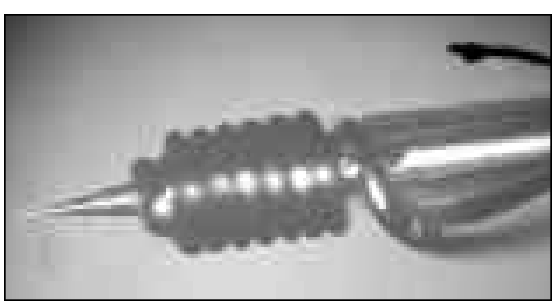

Figure. The micro-manipulator (microcoil and microtip), which measures $1 \mathrm{~mm}^{3}$ in size, offers an alternative to the optical tweezers method. The micron-scale coil diameter and ferromagnetic tip allow these electromagnetic tweezers to be fabricated with dimensions that are much smaller than traditional magnetic tweezers.

between the capillary and air, light was confined to the capillary due to total internal reflection, and magnetically trapped particles were clearly viewed without scattering interference. Furthermore, the magnetic samples were suspended in a liquid solution, and thus the capillary provided a container that both held the liquid and prevented the microtip from contacting the sample.

In their experiment, the researchers prepared an area on the sample that contained two closely spaced $(10-\mu \mathrm{m})$ PS beads but no magnetic particles. A nearby magnetic particle was located and, using the manipulator with a $100-\mathrm{mA}$ microcoil current, this bead was moved such that it traced out a figure eight pattern around the PS beads, thereby demonstrating submicron positioning of a micron-sized particle. The researchers estimated that approximately $0.5 \mathrm{pN}$ of force is exerted on the particle per $10 \mathrm{~mA}$ of current through the manipulator's microcoil. Coil currents up to $200 \mathrm{~mA}$ have been used without instrument degradation, indicating that forces as large as $10 \mathrm{pN}$ could be applied to particles. Even at these higher currents, the low $(1 \Omega)$ coil resistance ensures that sample heating does not occur.

"Our technique provides an attractive alternative to the optical tweezers method used in biological studies," said Barbic. "The micro-manipulator, which measures only $1 \mathrm{~mm}^{3}$ in size, could prove to be a valuable low-cost engineering tool. The device also has potential for further miniaturization and integration into micro-fluidics and MEMS systems."

STEFFEN K. KALDOR

\section{Switchable SQUID Fabricated in Loop Geometry from \\ $\mathrm{Fe}_{0.1} \mathrm{Co}_{0.9}$ and $\mathrm{Pb}$}

J. Eom and Mark Johnson at the U.S. Naval Research Laboratory have fabricat- 